K.A. van der Hucht, G. Koenigsberger \& P.R.J. Eenens, eds.

\title{
Wolf-Rayet stars as tracing the AGN-starburst connection
}

\author{
Daniel Kunth \\ Institut d'Astrophysique de Paris, France \\ Thierry Contini \\ European Southern Observatory, Garching, Germany
}

\begin{abstract}
We stress the importance of Wolf-Rayet stars for the understanding of the AGN phenomenon in galaxies. WR stars provide an unique opportunity to explore from the ground whether non-thermal nuclear activity and circumnuclear starbursts are connected. We review the known reported WR signatures observed so far in AGNs and point out some intrincacies related to the analysis of the spectra, linked to reddening correction, the origin of the $\mathrm{H} \beta$ line, etc. Finally, we advocate that integral field spectroscopy is a very promising tool to study this problem and present preliminary results of a long-term project that have been obtained at the CFHT in 1998.
\end{abstract}

\section{Introduction}

The nature of the dominant ionizing source in Active Galactic Nuclei is still poorly understood. Under the unified scenario, Seyfert 1 and Seyfert 2 galaxies represent the same kind of objects seen from different angles by the observer. In Seyfert 1 galaxies, one sees the central engine (a black hole surrounded by an accretion disc) whereas in Seyfert 2 galaxies a dusty torus completely blocks the non-thermal phenomenon. On the other hand, it has been suggested that starbursts may play an important role in the Seyfert phenomenon. In this case, AGNs are various distinct evolutionary phases of a burst of star formation in the core of early-type spiral galaxies. Physical processes associated with the starburst phenomenon can in principle account for most of the AGNs properties. In an hybrid version that take advantage of both views, a very compact circumnuclear starburst coexists with a massive central black hole responsible for the ionizing continuum. But a fundamental question remains to be answered. Are the non-thermal nuclear activity and the circumnuclear starbursts connected?

The question of the origin of the ultraviolet (UV) continuum in Seyfert 2 galaxies has recently received new attention. The advantage in chosing Seyfert 2 galaxies for further investigation of the AGN-starburst phenomenon is that one is not hampered by the central source. Early studies of IUE spectra by Heckman et al. (1995) showed that the UV radiation of Seyfert 2 galaxies is not compatible with the scenario of radiation scattered from an obscured nucleus. Instead its shape was fully understandable as being produced by a reddened starburst. HST has revealed that the UV continuum is spatially-resolved. Moreover, four bright Seyfert 2 galaxies have spectra dominated by the clear spectroscopic signatures of a starburst population. Strong stellar wind features were indeed detected ( $\mathrm{N} \vee \lambda 1240$, [Si IV $\lambda 1400$, and C IV $\lambda 1549)$ as well as stellar photospheric lines 
(Heckman et al. 1997; González-Delgado et al. 1998). In these four Seyfert 2 galaxies, selected on the basis of their UV brightness, the data provide evidence for the existence of nuclear starbursts. These account for a large fraction of the total intrinsic luminosity of the entire galaxy.

\section{The Wolf-Rayet population in emission-line galaxies}

\subsection{WR signatures in AGNs}

UV studies have opened new ways of studying the possible presence of a powerful starburst in AGNs. However, UV-bright Seyfert 2 galaxies are not so numerous, mostly because of dust obscuration, hence detailed UV observations are difficult to achieve even with the HST. This is why we believe that WR stars are excellent tracers to follow up in relation with the AGN-starburst phenomenon, mainly because they provide, in turn, conspicuous signatures in the visible. The detection of the broad emission feature at He II $\lambda 4686$ is indeed attributed mainly to WR stars of WN subtype, whereas C IV $\lambda 5808$, the strongest emission line of WC stars, is now currently observed in WR galaxies with modern CCD detectors (Schaerer, Contini \& Kunth 1999). Moreover, although they represent only a special short-lived phase in the evolution of the starburst population, of the star formation event (short in time interval) their occurence is highly expected in metal-rich environments.

Mrk 477 is the best case of a Seyfert 2 galaxy where the possible signature of massive WR stars has been detected (Heckman et al. 1997). But, because the broad stellar feature around $4686 \AA$ is very faint, it is difficult to separate this component from the bright He II $\lambda 4686$ nebular emission-line. The narrow nebular contribution can, in principle, be removed but illustrates the difficulty of detecting the WR features around $4700 \AA$. For this reason, the WR feature at $\mathrm{C}$ IV $\lambda 5808$ would be a better tool to detect massive stars in AGNs because it is free from unwanted nebular contributions (see sect. 3.2.). Mrk 477 has a very blue UV continuum, and a compact dusty starburst of a few hundred parsecs dominates the light from the UV to the near infrared. The bolometric luminosity of this nuclear starburst is $4 \times 10^{4} \mathrm{~L}_{\odot}$, a significant fraction of the total bolometric luminosity of Mrk 477. In the other three Seyferts (NGC 7130, NGC 5135 and IC 3639) studied by González-Delgado et al. (1998), He II $\lambda 4686$ seems to be of nebular origin.

\subsection{WR galaxies, IRAS starburts and AGNs}

As pointed out by Contini (these Proceedings), about 140 WR galaxies are now cataloged in the last compilation of Schaerer, Contini \& Pindao (1999). From previous detailed studies of WR galaxies some complications have been identified that one must keep in mind for AGN studies.

One key issue is the method that one adopts to estimate the number of WR stars. A convenient way to quantify the WR and the $\mathrm{O}$ stellar populations is to use the He II $\lambda 4686 / \mathrm{H} \beta$ luminosity ratios (Kunth \& Sargent 1981; Vacca \& Conti 1992; Vacca 1994). This method has some possible drawbacks (see also Schaerer, these Proceedings). In Table 1, we report some results that have been derived for four types of galaxies: Mrk 477 (Seyfert 2), Mrk 309 (a LINER previ- 
ously cataloged as Seyfert 2, then as a WR galaxy), NGC 6764 (first known as a Seyfert 2, then a WR galaxy and now reported as a LINER) and IRAS 01003-2238, a massive dusty starburst galaxy. One may ask whether the WR phenomenon in AGNs is a scaled up version of what we observe in intrinsically fainter WR galaxies. Indeed as shown by Kunth \& Schild (1986, hereafter KS86), one finds that as the absolute luminosity of galaxies increases so does the metallicity of the galaxy hence the luminosity $L_{\mathrm{WR}}$ of the WR feature. Using Table 1 , we can check that $L_{\mathrm{WR}}$ nicely falls onto the right side of KS86's fig. 5 where they plot metal-rich galaxies with the strongest $L_{\mathrm{WR}}$ luminosities. How well estimated are the WR/O ratios? KS86 argue that if one uses $E(B-V)$ derived from the Balmer decrement one makes the implicit assumption that all massive stars are deeply nested inside the nebular complex. Mas-Hesse \& Kunth (1999) have shown that the extinction obtained from the Balmer decrement is larger than the one derived from the UV continuum. This implies that the WR feature should be dereddened with a different correction value than the $\mathrm{H} \beta$ line and lead to a much smaller WR number than what is reported in Table 1 . Another concern is the use of the $\mathrm{H} \beta$ line which is also produced by the non-thermal central source and not only by high-mass stars as pointed out by Heckman et al. (1997), explaining why the $\mathrm{WR} / \mathrm{H} \beta$ flux ratio is not as large in Mrk 477 as in the less active galaxies.

Table 1. WR population in massive emission-line galaxies

\begin{tabular}{lcccc}
\hline galaxy & Mrk 477 & Mrk $309^{1}$ & NGC 6764 & IRAS 01003-2238 \\
& & & & \\
\hline morphology & compact & $\mathrm{Sa}$ & $\mathrm{SBbc}$ & $\ldots$ \\
$v_{\text {rad }}\left(\mathrm{km} \mathrm{s}^{-1}\right)$ & 11494 & 12831 & 2637 & 35328 \\
$M_{\text {abs }}$ & -21.0 & -21.7 & -21.1 & $\ldots$ \\
He II $\lambda 4686 / \mathrm{H} \beta$ & 0.10 & 0.67 & 0.43 & 0.61 \\
$E W(\mathrm{He}$ II $\lambda 4686)$ & $12 \AA$ & $8 \AA$ & $9 \AA$ & $\ldots$ \\
$E(B-V)$ & 0.23 & 0.7 & $\ldots$ & $\ldots$ \\
$\left.L_{\mathrm{WR}}(\operatorname{ergs~s})^{-1}\right)$ & $5 \times 10^{40}$ & $5 \times 10^{41}$ & $\ldots$ & $10^{41}$ \\
$N_{\mathrm{WR}}$ & $3 \times 10^{4}$ & $>10^{5}$ & $3 \times 10^{3}$ & $\ldots$ \\
WR/O & 0.4 & $\ldots$ & $\ldots$ & $\ldots$ \\
$12+\log (\mathrm{O} / \mathrm{H})$ & $\ldots$ & 8.90 & 8.88 & \\
\hline
\end{tabular}

References to Table 1: (1) Osterbrock \& Cohen (1982); (2) Armus et al. (1988)

\section{Integral field spectroscopy of nearby Seyfert 2 galaxies}

\subsection{Motivation}

We started a long-term project to clearly establish a direct link between the population of massive stars formed during a starburst and the AGN phenomenon. Indeed, despite the promising results obtained by Heckman and his collaborators (these Proceedings), fundamental questions remain. Is Mrk 477 an exception among Seyfert 2 galaxies? Where precisely is the starburst located, which has 
been detected by Heckman et al. (1997) in Mrk 477: exactly in the nucleus or in the circumnuclear region? Indeed, because of its large distance ( $153 \mathrm{Mpc})$, Heckman et al. were not able to locate the massive stars at a resolution better than $\sim 1 \mathrm{kpc}$, which is insufficient to assert that the starburst is nuclear.

The main goal of our program is to detect the spectral signatures of an important population of massive WR stars in the nucleus of Seyfert 2 galaxies. From the lists of Heckman et al. (1995), Terlevich et al. (1990), and GonzálezDelgado et al. (1997), we selected a dozen Seyfert 2 galaxies with very blue spectra. The advantage of this sample is to focus only on the nearest AGNs, located at a maximum distance of $\sim 60 \mathrm{Mpc}$ (with $\mathrm{H}_{0}=75 \mathrm{~km} \mathrm{~s}^{-1} \mathrm{Mpc}^{-1}$ ). Using MOS-ARGUS on the $3.6 \mathrm{~m} \mathrm{CFHT} \mathrm{with} \mathrm{a} \mathrm{FoV} \simeq 12^{\prime \prime} \times 7^{\prime \prime}$ and a spatial sampling of $0.4^{\prime \prime} /$ fiber, it is thus possible to explore the central region of galaxies with a resolution of $\sim 100 \mathrm{pc}$ (assuming a seeing of $0.6^{\prime \prime}$ ).

\subsection{Preliminary results}

Bi-dimensional spectroscopic observations of five galaxies (see Table 2) were obtained on the nights of 1998 May 31 - June 2 at CFHT. The data were acquired with ARGUS, a special mode for integral field spectroscopy with MOs. We used the blue grism B600 which gives a spectral coverage of about $3600-7200 \AA$ with a resolution of $\sim 4 \AA$. The total integration time for each galaxy ranges from 90 to $150 \mathrm{~min}$ in order to achieve a $\mathrm{S} / \mathrm{N}$ greater than 30 around 4700 and $5800 \AA$. The data reduction is in progress but preliminary results already illustrate the feasibility of this program.

Table 2. Wolf-Rayet features in nearby Seyfert 2 galaxies

\begin{tabular}{lccl}
\hline galaxy & morphology & $d(\mathrm{Mpc})$ & WR features \\
& & & \\
\hline & & & \\
NGC 6764 & SBbc & 35 & N III $\lambda 4640$, He II $\lambda 4686$, C III $\lambda 5696$, and C IV $\lambda 5808$ \\
NGC 5427 & SBc & 35 & N III $\lambda 4640$, He II $\lambda 4686$ (narrow), and C IV $\lambda 5808 ?$ \\
NGC 4388 & Sb & 33 & He II $\lambda 4686$ (narrow), and C IV $\lambda 5808 ?$ \\
NGC 5953 & S0-a & 26 & $\ldots$ \\
NGC 6951 & SABbc & 19 & $\ldots$ \\
\hline
\end{tabular}

In Fig. 1, we show the nuclear spectra of NGC 6764 and NGC 5427, two Seyfert 2 galaxies where we detected the spectral signature of WR stars. NGC 6764 is the best example in which broad emission lines from both WN and WC stars are clearly observed. Whereas the blue WR bump ( $\mathrm{N}$ III $\lambda 4640$ and He II $\lambda 4686$ ) was already known from previous long-slit observations (Osterbrock \& Cohen 1982; Eckart et al. 1996), we detect for the first time WR features in the red part of the spectrum (C III $\lambda 5696$ and C IV $\lambda 5808$ ), indicating an important population of massive WC stars in this object. Moreover, the $\mathrm{C}$ III $\lambda 5696$ broad emission line, which is rarely observed in WR galaxies (only two cases so far: NGC 1365, Phillips \& Conti 1992; and NGC 3049, Schaerer, Contini \& Kunth 1999), indicates a substantial population of late-type WC stars as expected in a high- 

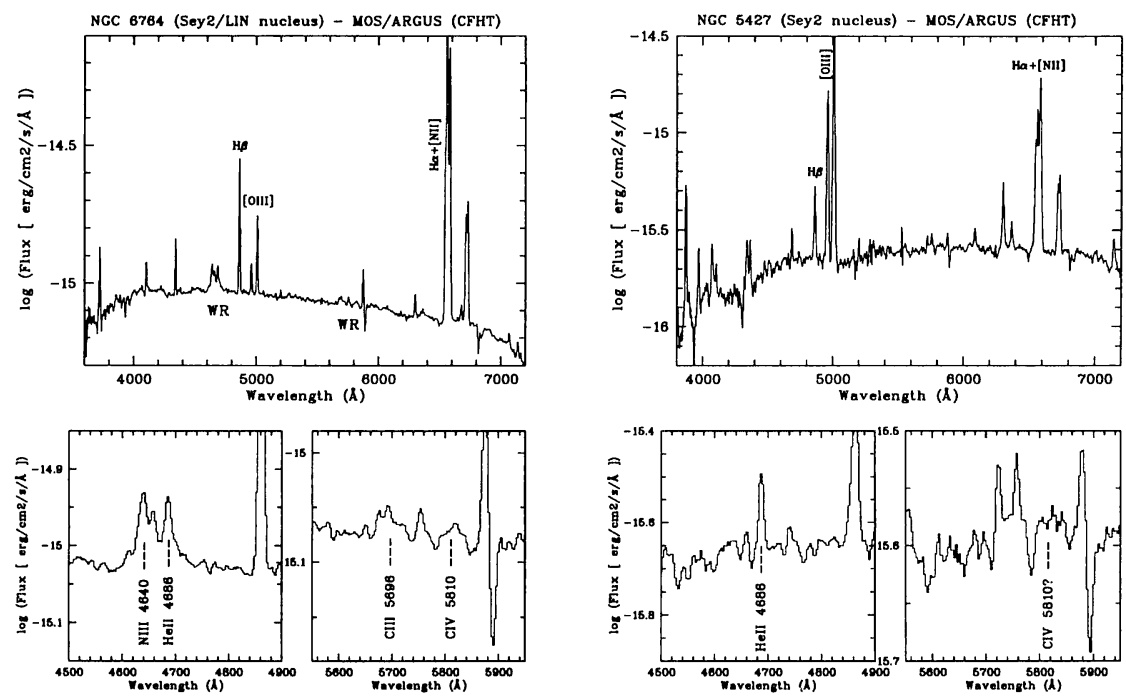

Figure 1. Nuclear spectra of two nearby Seyfert 2 galaxies observed with MOS-ARGUS at CFHT. NGC 6764 (left) represents the best case of an AGN where the spectral signatures of both WN ( $\mathrm{N}$ III $\lambda 4640$ and He II $\lambda 4686$ ) and WC stars (C III $\lambda 5696$ and $\mathrm{C}$ IV $\lambda 5808)$ are clearly detected, indicating a young and massive starburst in the core of this AGN. The detection of WR stars in the nucleus of NGC 5427 (right) is less clear. The blue WR bump is contaminated by the bright nebular He II $\lambda 4686$ emission line and the $s / n$ in the red part of the spectrum is too low to see clearly the C IV $\lambda 5808$ line.

metallicity environment. As can be seen in Fig. 1, the blue WR bump around $4700 \AA$ is usually contaminated by the bright and narrow nebular He II $\lambda 4686$ line which is very common in Seyfert 2 spectra. It is thus very difficult to derive an accurate number of WR stars from the broad He II $\lambda 4686$ component. Instead, it is better to use the "red" WR features around $5800 \AA$ because these broad lines are free of any contamination by close nebular emission lines.

The detection of WR spectral features is more ambiguous in the Seyfert 2 galaxies NGC 5427 (see Fig. 1) and NGC 4388. The blue part of their spectrum around $4700 \AA$ is dominated by the bright nebular He II $\lambda 4686$ emission line and the $\mathrm{S} / \mathrm{N}$ around $5800 \AA$ is too low to clearly identify the broad C IV $\lambda 5808$ line. No WR features have been found in the two remaining galaxies, NGC 5953 and NGC 6951.

A complete reduction and analysis of the bi-dimensional spectra will give us crucial information on the AGN-starburst connection in these galaxies. By producing a map of the WR spectral features, we should be able (1) to distinguish between a nuclear starburst and circumnuclear star-forming regions, and (2) to estimate the number of massive stars and their contribution to the ionizing and bolometric luminosity of the AGN. 


\section{Conclusions}

WR stars are excellent tracers to follow up in relation with the AGN-starburst phenomenon, mainly because they provide conspicuous signatures in the visible. Bi-dimensional spectroscopic observations are currently performed, with preliminary results illustrating the feasibility of such a program. Spectral features from both WN and WC stars are clearly seen in the nucleus of the Seyfert 2 galaxy NGC 6764. An important result is the detection of the C III $\lambda 5696$ broad emission line originating from late-type WC stars, which are rarely observed in WR galaxies but are expected to be present in a high-metallicity environment, such as an AGN.

\section{References}

Armus, L., Heckman, T.M., Miley, G.K. 1988, ApJ 326, L45

Eckart, A., et al. 1996, ApJ 472, 588

Heckman, T.M., et al. 1995, ApJ 452, 549

Heckman, T.M., et al. 1997, ApJ 482, 114

González-Delgado, R.M., et al. 1997, ApJS 108, 155

González-Delgado, R.M., et al. 1998, ApJ 505, 174

Kunth, D., Sargent, W.L.W. 1981, A\&A 101, L5

Kunth, D., Schild, H. 1986, A\&A 169, 71

Osterbrock, D.E., Cohen, R.D. 1982, ApJ 261,64

Mas-Hesse, J.M., Kunth, D. 1999, A\&A in press (astro-ph/9812072)

Phillips, A.C., Conti, P.S. 1992, ApJ 395, L91

Schaerer, D., Contini, T., Pindao, M. 1999, A\&AS 136, 35

Schaerer, D., Contini, T., Kunth, D. 1999, A\&A 341, 399

Terlevich, E., Diaz, A.I., Terlevich, R. 1990, MNRAS 242, 271

Vacca, W.D. 1994, ApJ 421,140

Vacca, W.D., Conti, P.S. 1992, ApJ 401, 543

\section{Discussion}

Marchenko: This is in regard to WR galaxies with suggested large numbers of WR stars. If they have $\sim 10^{5}$ WR stars, one would expect roughly one SN explosion every 5 years. Are there any statistics about SN explosions in WR galaxies with such large numbers of WR stars?

Kunth: Unfortunately not. Monitoring should be conceivable since a new catalogue of WR galaxies is now available (Schaerer et al. 1999) that contains more than hundred galaxies.

Maeder: WR stars are expected to give SN explosions which are faint in the visible (but not in gamma- and X-rays) because there is no large envelope to thermalize the shock.

Leitherer: What is the output of light with respect to the AGN light in the sample you observed?

Kunth: I guess the starburst emits a significant fraction (50\%?) of the UV light. 
Cid-Fernandes: I would just like to emphasize that all WR-Seyfert 2 galaxies known are selected because they are UV bright (e.g., Markarian galaxies), which obviously favors detection of young starbursts. It will be nice to know whether the nucleus is still active when the starburst ages to say $10^{8}$ years.

Schaerer: Can you say something about the spatial distribution of the massive stars you detect?

Kunth: Not yet because we are in a preliminary stage of the analysis but this is precisely what we want to look at.

Niemela: You may consider that also accretion discs e.g., massive X-ray binaries, cataclysmic variables, etc., show WR type spectra, and those WR lines in AGN may come from a massive accretion disc.

Kunth: This must be considered indeed.

van der Hucht: You are concerned by the large number of WR stars $\left(>10^{5}\right)$ in some particular starburst galaxies. I assume your WR number-estimates are based on 'regular' WR luminosities. In NGC 3603 and R 136a superluminous WN6 stars have been found. Also WR 22 is superluminous by a factor $\sim 5$ or so. Assuming that your starburst have superluminous WN6 stars, would bring the numbers down by a factor $\sim 5$, and thus also the WR/O ratio.

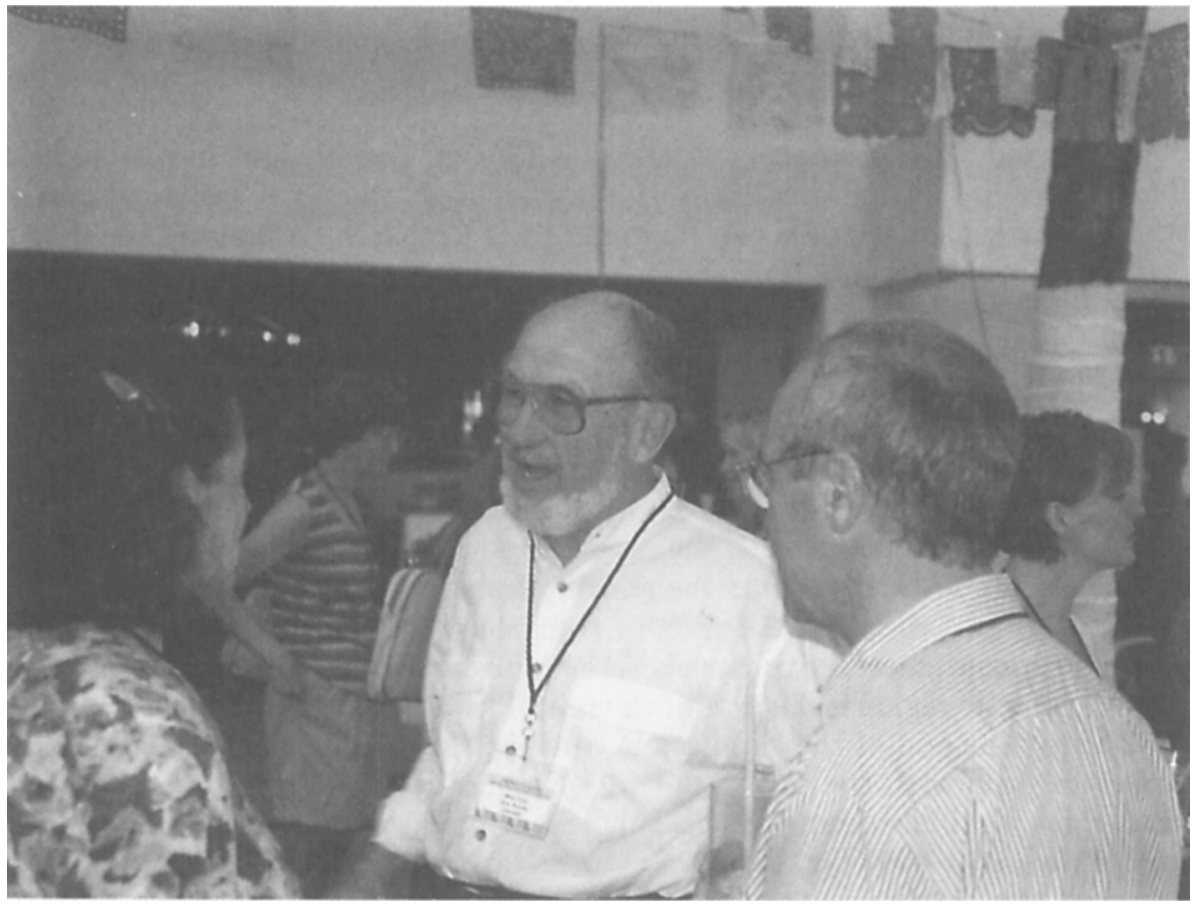

